\title{
Amt auf Zeit. Eine explorative Studie zum beruflichen und politischen Verbleib ehemaliger Bundestagsabgeordneter
}

\author{
Maria Kreiner
}

In der Literatur zur Elitenforschung wurde immer wieder festgestellt, dass Studien über den Verbleib von ausgeschiedenen Berufspolitikern fehlen. ${ }^{1}$ Der Stand der Forschung zeigt, dass bislang vor allem der Zugang zum politischen Amt, seine Ausübung, die Einstellungen der Amtsträger und der Verlauf ihrer politischen Karrierewege erforscht wurden. Die Frage, was nach dem Ausscheiden aus der Politik geschieht, wurde zwar vereinzelt aufgeworfen und auch als Teilfrage eines übergeordneten Kontextes behandelt, bildete aber nie den ausschließlichen Forschungsgegenstand. ${ }^{2}$ Im Folgenden wird dieses Feld besser ausgeleuchtet. Aus Interviews mit 38 ehemaligen Bundestagsabgeordneten konnten Eindrücke über die berufliche und politische Situation von Parlamentariern nach dem Mandat gewonnen werden. So entsteht zum einen ein anschauliches Bild aus Einzelfällen, und zum zweiten können systematische Hypothesen für weitere (repräsentative) Forschungen generiert werden.

Zwischen März 2003 und März 2004 wurden Abgeordnete, die 1994 oder 1998 aus dem Bundestag ausgeschieden waren, in einem persönlichen Gespräch von der Verfasserin befragt. ${ }^{3}$ Anhand eines Leitfadens wurde der berufliche und politische Werdegang vor, während und nach dem Bundestagsmandat erfasst. Dabei bildete der berufliche Verbleib nach dem Mandatsende den Schwerpunkt der Befragung. Ferner wurde erhoben, ob für den Fall des Ausscheidens aus dem Amt Vorsorge getroffen worden war und wodurch die persönliche Situation nach dem Bundestagsmandat am stärksten gekennzeichnet war. Hier werden die Ergebnisse des beruflichen und politischen Verbleibs zusammengefasst dargestellt. ${ }^{4}$

1 Vgl. Klaus von Beyme, Die politische Elite in der Bundesrepublik Deutschland, München 1971, S. 147; Rolf-Peter Lange, Strukturwandlungen der westdeutschen Landesregierungen 1946-1973. Das politische Führungspersonal der Bundesländer: Eine empirische Studie zur Soziologie der Herkunft, Rekrutierung und Zirkulation der westdeutschen Landesregierungen, Diss., Berlin 1976, S. 402; Jens Borchert / Klaus Stolz, Die Bekämpfung der Unsicherheit: Politikerkarrieren und Karrierepolitik in der Bundesrepublik Deutschland, in: PVS, 44. Jg. (2003), H. 2, S. 148 173, S. 155 Fußnote 10 und S. 168 Fußnote 22.

2 Zum Stand der Forschung siehe Maria Kreiner, Amt auf Zeit. Eine Verbleibsstudie über ehemalige Bundestagsabgeordnete, Baden-Baden 2006, S. $15 \mathrm{ff}$.

3 Der Eingrenzungsprozess von der Grundgesamtheit zur Untersuchungsgruppe erfolgte in vier Schritten. Zunächst wurde die Gesamtheit der noch lebenden ehemaligen Bundestagsabgeordneten (1282 Personen, Stand 18. Juli 2002) auf die Ausgeschiedenen der 12. und 13. Wahlperiode eingegrenzt (368 Personen). Daraus wurden in einem zweiten Schritt die Personen selektiert, die nach dem Ausscheiden aus dem Bundestag noch nicht das Pensionsalter erreicht hatten (212 Personen). Von diesen Personen wurden in einem dritten Schritt Kontaktmöglichkeiten recherchiert (134 Adressen konnten ausfindig gemacht werden). Im vierten Schritt wurden für die Erhebung Personen, von denen eine Kontaktadresse vorlag, in drei Wellen angeschrieben (insgesamt 76 Personen). Von den Angeschriebenen haben sich 40 Personen bereit erklärt, ein Interview zu geben; letztlich wurden 38 Interviews realisiert.

4 Weitere Ergebnisse, nähere Angaben über den Untersuchungsablauf, die Methode und den Interviewleitfaden finden sich in Maria Kreiner, a.a.O. 


\section{Beruflicher Verbleib ehemaliger Bundestagsabgeordneter}

Zunächst stellt sich die Frage, welchen Berufsgruppen die Befragten angehören, um im Folgenden den beruflichen Verbleib und spätere Aussagen besser einordnen zu können. Zusätzlich werden die Berufsgruppen nach Ost- beziehungsweise Westherkunft aufgeschlüsselt (vgl. Tabelle 1).

\begin{tabular}{|c|c|c|c|}
\hline \multicolumn{4}{|c|}{$\begin{array}{l}\text { Tabelle 1: Befragte ehemalige Bundestagsabgeordnete nach Berufsgruppen und nach Ost- } \\
\text { und Westherkunft (absolut) }\end{array}$} \\
\hline Berufsgruppe & $\begin{array}{l}\text { Befragte aus Ost- } \\
\text { deutschland }\end{array}$ & $\begin{array}{l}\text { Befragte aus West- } \\
\text { deutschland }\end{array}$ & insgesamt \\
\hline Beamte & - & 5 & 5 \\
\hline Öffentlicher Dienst & 2 & 3 & 5 \\
\hline Organisationen & 6 & 2 & 8 \\
\hline Wirtschaft & 2 & 6 & 8 \\
\hline Selbständige & - & 3 & 3 \\
\hline Freie Berufe & 1 & 2 & 3 \\
\hline Hausfrauen & 1 & 4 & 5 \\
\hline Arbeiter & 1 & - & 1 \\
\hline insgesamt & 13 & 25 & 38 \\
\hline
\end{tabular}

Aus den Untersuchungsergebnissen lassen sich hinsichtlich des beruflichen Verbleibs der Befragten folgende Fallgruppen bilden:

(1) Berufsrückkehrer: die Befragten, die ihre Berufstätigkeit mit dem Eintritt in den Bundestag aufgegeben haben und nach dem Mandatsende wieder in ihre vorherige Berufstätigkeit eingestiegen sind. Es handelt sich um insgesamt drei Personen (9,4 Prozent der 32 Befragten, die vor dem Mandatsantritt erwerbstätig waren).

(2) Berufswechsler: jene zehn Befragten (31,2 Prozent), die ihre Berufstätigkeit mit dem Eintritt in den Bundestag aufgegeben haben und nach dem Mandatsende eine andere Berufstätigkeit ausübten. Diese Gruppe kann weiterhin differenziert werden in jene Personen, die im politischen Bereich geblieben sind (Landtagsmandat, Staatssekretär; zwei Befragte) und solche, die in den außerpolitischen Bereich wechselten (acht).

(3) Berufsfortführer: Befragte, die ihre Berufstätigkeit während der Mandatszeit parallel fortgeführt beziehungsweise auf ein Minimum reduziert haben und nach dem Mandatsende weiterhin fortführten beziehungsweise in vollem Umfang wieder aufnahmen. Dieser Gruppe gehören insgesamt acht Personen (25,0 Prozent) an.

(4) Pensionäre: dazu zählen die Befragten, die nach dem Mandatsende in Frühpension gingen (sieben Personen, 21,9 Prozent). Diese Gruppe sollte allerdings gar nicht in der Untersuchung berücksichtigt werden, weshalb ihr Vorkommen hier nur zufällig ist. ${ }^{5}$

5 Da der berufliche Verbleib nur bei den ehemaligen Abgeordneten relevant ist, die nach dem Ausscheiden aus dem Bundestag noch nicht das Pensionsalter erreicht haben, wurde die Gruppe der Pensionäre aus der Untersuchung ausgeklammert. Da aber aus den offiziellen Angaben nicht ersichtlich ist, ob ein vorzeitiger Pensions- beziehungsweise Rentenanspruch aus der vorherigen beruflichen Tätigkeit besteht, ob die Person aufgrund von Krankheit die Erwerbsunfähigkeitsrente beziehungsweise Frühpension beantragt hat, oder ob die Person privat so vermögend ist, dass 
(5) Problemfälle: Befragte, die ihre Berufstätigkeit mit dem Eintritt in den Bundestag aufgegeben haben und nach dem Mandatsende zeitweilig oder dauerhaft arbeitslos wurden. Dies betrifft insgesamt vier Personen (12,5 Prozent).

\subsection{Berufsrückkehrer, Berufswechsler, Berufsfortführer}

Nur drei, also die wenigsten der 26 abhängig beschäftigten Befragten ${ }^{6}$ sind zur vorherigen Stelle zurückgekehrt (Berufsrückkehrer), obwohl fast die Hälfte von ihnen (zwölf) eine Rückkehrmöglichkeit und damit eine Arbeitsplatzgarantie hatte. Die Berufswechsler bilden mit zehn Personen die größte Fallgruppe. Dies erklärt sich zum einen damit, dass sich die meisten der Befragten ohne Rückkehrmöglichkeit zur vorherigen Stelle nach dem Mandatsende um eine neue Arbeitsstelle bemühten und folglich nicht zu ihrem vorherigen Arbeitsplatz zurückgekehrt sind. Zum anderen nutzten die befragten Beamten und Angestellten des öffentlichen Dienstes ihre Rückkehrmöglichkeit nicht, sondern sie konnten sich auf der Basis dieser Arbeitsplatzgarantie beruflich neu orientieren und etablieren. Diese Gelegenheit wurde von nahezu allen befragten Beamten und Angestellten des öffentlichen Dienstes wahrgenommen. Der vorwiegende Grund hierfür war ihre geringe Motivation, sich wieder in ihr vorheriges Berufsfeld zu integrieren ${ }^{7}$; stattdessen wollten sie ihre im Bundestag erworbenen Kenntnisse und Fähigkeiten nach dem Mandatsende beruflich nutzen. Am Beispiel einer befragten Person der Gruppe Beamte:

„Ich wusste, es ist der Vorteil - das macht einen auch so sicher - ich hatte als beurlaubter Beamter den Anspruch wieder zurückzukehren ... . Die Rosinen, die ich mir rauspicken [konnte], ... waren dann Stellenanzeigen. ... Ich habe zunächst ... einen Lehrauftrag angenommen. Das war wunderbar: im November hörte das Mandat auf, Ende Oktober habe ich an der hiesigen Universität einen Lehrauftrag in Politikwissenschaft vertretungsweise wahrgenommen für einen Professor, der Forschungssemester machte." (I.17.W.k) ${ }^{8}$

Fragt man nach den Gründen für den Wechsel der Berufstätigkeit der befragten Beamten und Angestellten des öffentlichen Dienstes, kommt zutage, dass es für sie wenig reizvoll gewesen wäre, wieder ihre vorherige berufliche Tätigkeit auszuüben:

„Ich habe gern unterrichtet, bin gerne in die Schule gegangen. Ich würde sagen, dass ich heute froh bin, nicht mehr in der Schule zu sein. Ich weiß ganz genau, dass ich oft gesagt

sie bis zum Pensionsalter nicht auf Erwerbsarbeit angewiesen wäre, befanden sich unter den Angeschriebenen auch Pensionäre, was sich erst während der Interviews herausstellte.

6 Zu den abhängig Beschäftigten zählen Beamte und Angestellte des öffentlichen Dienstes, welche aufgrund des Rückkehrrechtes über eine Arbeitsplatzgarantie verfügen und Angestellte politischer und öffentlicher Organisationen sowie Angestellte in der Wirtschaft, die in der Regel keine Rückkehrmöglichkeit zur vorherigen Arbeitsstelle haben.

7 Adalbert Hess weist darauf hin, dass das Abgeordnetenmandat für den weisungsgebundenen Beamten „ein beachtlicher sozialer Aufstieg mit größerer Gestaltungsfreiheit, besonderen Privilegien und - nicht selten - mit Chancen, eine höhere berufliche Ebene zu erreichen", ist. Adalbert Hess, Politikerberufe und Politiker - Betrachtungen zur Parlamentssoziologie, in: ZParl, 16. Jg. (1985), H. 4, S. $581-587$, S. 583.

8 Der Code hat folgende Bedeutung: „““ steht für Interview; „Zahl“ gibt die Nummer des Interviews an (1-38); „W“ beziehungsweise „O “ steht für die West- oder Ostzugehörigkeit des Befragten; „, $\mathrm{k}$ “ bedeutet, dass der Befragte kandidierte, aber nicht wieder gewählt wurde; „nk“ bedeutet, dass der Befragte nicht wieder kandidierte. Angaben wie sie Parteizugehörigkeit, das Geschlecht oder das Jahr des Ausscheidens wurden nicht codiert, um die Anonymität zu wahren. 
habe: ,Ich gehe gerne in die Schule, hat Spaß gemacht'. [...] Ich hatte inzwischen durch dieses Mandat andere Horizonte - oder Blut geleckt, hatte andere Interessen, als nun den ,Subjonctif' in Französisch da zu üben oder zum x-ten Mal die Grundlagen der Demokratie mit knapp Wahlberechtigten durchzukauen. Wenn man so will, Schule war nicht mehr so interessant." (I.17.W.k)

Andere waren hingegen schon vor dem Mandatsantritt mit ihrer Beamtentätigkeit unzufrieden und fühlten sich unterfordert:

„Also, Beamter hab’ ich ja nur aus rationellen Gründen gemacht. Das ergab sich so, das war kein Lebenswunsch, sondern das war eine pragmatische Sache, dass ich [das] gemacht hab' und was ich machte, hat mich geistig nicht ausgefüllt. Man machte seine Tätigkeit, aber war geistig nicht gefordert. Interessiert hat mich die Politik. [...] Da hab' ich viel Zeit investiert ... Das war mein Hobby und war eigentlich auch so mein Lebensziel und das, was mich ausfüllte. Insofern hab' ich [meine Dienststelle] nicht vermisst. Sondern das war so ein Job, um den Lebensunterhalt zu verdienen." (I.14.W.k)

Die Unterforderung und Langeweile am Arbeitsplatz fand in vielen Äußerungen ihren Ausdruck. Auf die Frage, ob die beruflichen Pläne mit der Aussicht auf das Bundestagsmandat kollidierten, antwortete ein ehemaliger Angestellter des öffentlichen Dienstes: „Nee, nee, überhaupt nicht. Ich wollte ja ohnehin aus dem Ministerium weg. Entweder ins Parlament oder in die freie Wirtschaft. Ich fühlte mich zu wenig gefordert." (I.35.W.k) Die Person mutmaßte, dass wohl die wenigsten ausgeschiedenen Abgeordneten wieder zu ihrer vorherigen Stelle zurückkehren würden, denn „selbst wenn man ein Rückkehrrecht hat, was wollen Sie denn noch als Referatsleiter in einem Ministerium? Sie waren Abgeordneter!“ (I.35. W.k)

Eine weitere Gruppe ist die der Berufsfortführer. Nur zwei der abhängig beschäftigten Befragten blieben während der Mandatszeit berufstätig. Die befragten Selbständigen (drei Personen) und Angehörigen freier Berufe (drei Personen) hingegen versuchten, ihre Berufstätigkeit mit dem Bundestagsmandat zu vereinbaren, denn keiner von ihnen wollte sein Unternehmen oder seine Kanzlei für ein Amt auf Zeit aufgeben. Dieser Umstand hatte zur Folge, dass sie entweder unter enormen physischen Belastungen ein berufliches Doppelleben führten oder ihre Berufstätigkeit auf ein Minimum reduzierten und ihre Geschäfte vornehmlich von Vertretern wahrnehmen ließen. Die folgende befragte freiberuflich tätige Person kapitulierte bald vor der Arbeitsbelastung durch zwei Vollzeitberufe und nahm einen Vertreter in die Kanzlei auf:

„Und wie ich dann in den Deutschen Bundestag gegangen bin, ließ sich natürlich das trotzdem notwendige persönliche Engagement im Anwaltsberuf einfach nicht herstellen. Das heißt, ich habe nur, um nicht aus den juristischen Alltagsdingen heraus zu kommen, mich mehr oder weniger gezwungen, Fälle zu machen und sie möglichst gut zu machen. Aber im Grunde genommen war man so schnell von der Politik vereinnahmt, dass man einfach den Beruf nicht mehr ausüben konnte. Ich bin Sozius geblieben, einer von zweien, später haben wir dann einen dritten Mann reingenommen." (I.32.W.k)

Die Möglichkeit, die Arbeitsstelle durch einen Stellvertreter zu besetzen, hatten die abhängig beschäftigten Befragten nicht. Die folgende Person aus der Gruppe Angestellte in der Wirtschaft gab letztlich das Mandat aufgrund der beruflichen Doppelbelastung auf:

„Das ist ja eigentlich einer der Gründe, warum ich wieder [aus dem Bundestag] ausgestiegen bin. Einen Betrieb leiten ... und Bundestagsabgeordneter zu sein, ist eigentlich nicht vereinbar. [...] Ich meine, wenn man so etwas übernimmt und sagt: ,Ich will in der Politik mitarbei- 
ten', dann muss man auch bereit sein, über das Maß hinaus arbeiten zu wollen. Da kann ich nicht sagen: ,Ich hab' einen Achtstundentag und den teil' ich noch auf.' Politik allein erfordert schon zehn oder zwölf Stunden Arbeit, und wenn jemand eben die Kraft hat und sagt: ,Ich kann jeden Tag noch drei oder vier Stunden für meinen Betrieb hinten dranhängen', dann soll er das machen. ... Ob er es dauerhaft so kann, ist eine andere Frage.“ (I.04.O.nk)

Als Grund für die Fortführung der Berufstätigkeit während der Mandatszeit gaben die entsprechenden Befragten insgesamt an, damit ihre Existenz nach einem etwaigen Mandatsverlust gesichert zu haben, was ihnen eine materielle Unabhängigkeit vom Mandat und Entscheidungsfreiheit gegenüber ihrer Fraktion ermöglichte. So antwortete eine Person auf die Frage, welches die sinnvollste Vorbereitung auf ein mögliches Ausscheiden aus dem Bundestag war: „Zu sagen: ,Die Firma läuft weiter'. Das war die sinnvollste [Vorbereitung]." (I.02.W.k) Eine andere Person hat sich während der Mandatszeit selbständig gemacht. Einer der Gründe dafür war die Vorsorge für das Ausscheiden aus dem Bundestag:

„Man sollte nicht am Politikersessel kleben, und man weiß nicht, wie es kommt. Es ist einfach eine Vorsorge gewesen, die mir zwar sehr viele Sorgen gemacht hat, weil man eigentlich zwei Sachen nicht machen kann, weil allein die Abgeordnetentätigkeit wahnsinnig war. Ich hatte hier einen Leiter eingesetzt, aber das lief eben nicht ganz so, als wenn man selber dran ist und selber eben Kredite bedienen muss. Und von daher lief es in diesen zwei Jahren, wo ich noch im Bundestag war, lief das hier nicht so glänzend. Das hat sich dann sofort auch geändert, als ich dann hier drin war." (I.01.O.k)

Eine weitere befragte Person betonte die Unabhängigkeit:

„Der Beruf war vor allem für mich die Garantie für meine Unabhängigkeit. Ich habe manchmal durchblicken lassen, mit dem Gedanken gespielt, gegen die Fraktion zu stimmen. Sieben- oder elfmal habe ich in namentlicher Abstimmung gegen die Fraktion gestimmt." (I.10. W.nk)

Diese Unabhängigkeit besaßen die befragten Beamten und Angestellten des öffentlichen Dienstes ebenso, allerdings ohne dafür parallel zum Mandat ihrer Berufstätigkeit weiterhin nachgehen zu müssen und ohne das Risiko, ihren Arbeitsplatz zu verlieren. Im Vergleich zu ihnen konnten nicht alle befragten Selbständigen und Angehörigen freier Berufe problemlos wieder in ihren Betrieb zurückkehren. Ein Selbständiger musste den Konkurs seines Unternehmens abwenden; die bereits oben zitierte Person musste sich als Anwalt eine neue Existenz aufbauen:

„Ich hatte erst mal große Schwierigkeiten gehabt mit meinem Sozius, der in der Zwischenzeit hier völlig aufgeräumt hatte. Ich bin wirklich unter die Räuber gefallen .. . Ich musste ganz vorne anfangen. Da gab es Monate, da habe ich einen Umsatz gemacht von vielleicht 25.000 Mark und hab' 300 Mark rausbekommen, weil die anderen alles abgesahnt haben.“ (I.32.W.k)

Die Existenz der Gruppe der Berufsfortführer kann als Reaktion der Berufspolitiker auf die existentiell bedrohliche Konstruktion ihres Mandates verstanden werden: Sie führen ihren Beruf als existentielle Sicherheit für die Zeit nach dem Mandatsende fort. ${ }^{9}$

9 Für Thesen zur Bekämpfung der Unsicherheit des politischen Hauptamtes vgl. Jens Borchert I Klaus Stolz, a.a.O. 


\subsection{Die Rolle des Übergangsgeldes bei der beruflichen Wiedereingliederung}

Für die Wiedereingliederung in das Berufsleben erhalten ausgeschiedene Bundestagsabgeordnete ein Übergangsgeld. Derzeit gilt, dass für jedes Jahr der Parlamentszugehörigkeit ein Monat Übergangsgeld in Höhe der jeweils aktuellen Abgeordnetenentschädigung ${ }^{10}$ gezahlt wird, nach einer Wahlperiode also für vier Monate, insgesamt aber längstens für $18 \mathrm{Mo-}$ nate. ${ }^{11}$ Diese Start- und Anpassungshilfe hat darüber hinaus den Zweck, die mit dem Ausscheiden aus dem Parlament verbundenen Kosten zu erstatten. ${ }^{12}$ „Da die Mitgliedschaft im Parlament grundsätzlich nur auf Zeit angelegt ist, müssen die Abgeordneten von vornherein damit rechnen, das Parlament nach Ablauf einer oder mehrerer Wahlperioden wieder zu verlassen, und es kann von ihnen erwartet werden, daß sie sich auf eine andere Lebensgrundlage nach Beendigung des Mandats einstellen."13

Die folgende befragte Person gründete mit Hilfe des Übergangsgeldes nach dem Mandatsverlust eine Existenz:

„Das Übergangsgeld habe ich genutzt, um mir die Existenz aufzubauen. Als ich .. damit begann, da musste ich mir erst mal gewissermaßen vom Schreibtisch alles wieder - na ja, im Wahlkreisbüro hatte ich noch einen Schreibtisch - aber man muss Investitionen machen, da ist nicht am ersten Tag gleich Geld geflossen. ... Also, das hat natürlich ein halbes bis dreiviertel Jahr gedauert bis ich wieder in das Geldverdienen reingekommen bin: Musste Partner finden, musste eine $\mathrm{GmbH}$ gründen, musste $\mathrm{GmbH}$-Einlagen machen, das kostet alles Geld und das habe ich zu einem beträchtlichen Teil aus meinem Übergangsgeld genommen. [...] Ich kann es Ihnen nur an meiner eigenen Vita sagen, ohne das Übergangsgeld - ja was hätte ich jetzt machen sollen? Also, wenn ich mir nichts auf die Seite gelegt hätte, wie auch immer, hätte ich dann ans Sozialamt klopfen müssen oder wie?" (I.34.W.k)

Eine Person, die nicht wieder für den Bundestag kandidierte, war zunächst vollständig auf das Übergangsgeld angewiesen, weil sie ein Mandat im Europäischen Parlament erringen wollte, was allerdings missglückt war. Sie fand dann aber bald eine Anstellung:

„[D] as [Übergangsgeld] hat mich erst mal in den Stand gesetzt, ruhig abzuwarten und zu sondieren, was ich nun weiter machen könnte. Ich war auf Arbeit angewiesen, weil ich ja

10 Mit Stand vom 1. Januar 2003 beträgt die Abgeordnetenentschädigung 7009 EUR brutto. Vgl. AbgG, $\$ 11$, Abs. 1 .

11 Vgl. ebenda. Zitiert aus: Deutscher Bundestag - Verwaltung (Hrsg.), Informationen für ausgeschiedene Abgeordnete, Berlin 2002, S. 6. Diese Bestimmung gilt seit 22. Dezember 1995. Das alte Recht (gültig seit 18. Februar 1977) sah vor, dass für jedes Jahr der Parlamentszugehörigkeit ein Monat Übergangsgeld in der Höhe des fiktiven Bemessungsbetrages von umgerechnet 5.301 Euro gezahlt wird. Dauerte die Parlamentszugehörigkeit länger als die Hälfte einer Wahlperiode kamen drei weitere Monate, ansonsten zwei weitere Monate hinzu. Das heißt, dass nach vier Jahren Parlamentszugehörigkeit sieben Monate Übergangsgeld in Anspruch genommen werden konnten. Die Anspruchsdauer betrug höchstens 36 Monate. Vgl. ebenda. Diese (alte) Bestimmung gilt für alle Befragten dieser Untersuchung, die nach der 12. Legislaturperiode ausgeschieden sind. Die Befragten, die am Stichtag des 22. Dezember 1995 Mitglied des Deutschen Bundestags waren, konnten sich bis zu ihrem Ausscheiden für eine Anwendung des alten oder des neuen Rechts entscheiden. Es ist zu vermuten, dass sich die meisten Betroffenen für die Geltung des alten Rechts entschieden haben, da seine Bestimmungen für ausgeschiedene Abgeordnete finanziell vorteilhafter sind.

$12 \mathrm{Vgl}$. Annette Fischer, Abgeordnetendiäten und staatliche Fraktionsfinanzierung in den fünf neuen Bundesländern, Frankfurt am Main u.a. 1995, S. 85.

13 Ebenda, S. 86. 
meinem Mann zugesagt hatte, für das Studium für eines unserer beiden Kinder aufzukommen, denn nie im Leben hätte ich mir träumen lassen, dass die Europakandidatur schief gehen könnte. [...] Für mich war es notwendig. Ich hatte keinen Beruf, ich war rausgeflogen und ich hätte nicht gewusst, wovon ich leben sollte." (I.29.W.nk)

Die berufliche Wiedereingliederung wurde allerdings nur bei knapp einem Viertel der Befragten mithilfe des Übergangsgeldes erreicht. Bei wenigen genügte die Anspruchsdauer nicht zum erfolgreichen Einstieg in das Erwerbsleben, weshalb sie arbeitslos wurden. Für die meisten der Befragten erfüllte das Übergangsgeld andere Zwecke als die berufliche Wiedereingliederung. Die entsprechenden Befragten fanden entweder sofort nach dem Mandatsende eine Anstellung oder gingen in den Ruhestand.

Bei ehemaligen Abgeordneten, die nach dem Mandatsende relativ bald wieder beziehungsweise nahtlos ein bezahltes öffentliches Amt übernahmen, wurde das Übergangsgeld, wie rechtlich vorgesehen, mit dem Gehalt verrechnet. ${ }^{14}$

Einige Angestellte des öffentlichen Dienstes nehmen nicht sofort nach dem Mandatsende wieder ihre Arbeit auf, sondern warten mit der Wiederbeschäftigung, bis das Übergangsgeld ausgelaufen ist:

„[A]lso, ich hab’ mich zurückgemeldet ..., habe aber erst mal das Übergangsgeld - weil das ja ein öffentliches Unternehmen ist und dementsprechend Übergangsgelder mit dem Gehalt wieder verrechnet werden - war für mich sozusagen der Reiz nur bedingt, gleich wieder arbeiten zu gehen, weil ich dann für lau gearbeitet hätte. Ich hab’ das also auslaufen lassen und mich dann wieder zurückgemeldet." (I.21.O.nk)

Befragte aus anderen Berufsgruppen, die nicht auf das Übergangsgeld angewiesen waren, setzten es für unterschiedliche Zwecke ein. Zum Beispiel stellte ein Angestellter in der Wirtschaft das Übergangsgeld seiner Familie zur Verfügung:

„Das habe ich unserem Sohn zur Verfügung gestellt und der Schwiegertochter. Der Sohn hatte gerade geheiratet, es kam ein Baby. ... Das Übergangsgeld ist für die zweckerfüllend, die jünger sind als ich - wir haben ja jetzt diesen stark verjüngten Bundestag - die vom Studium direkt in den Bundestag sind, die nie beruflich gearbeitet haben, die müssen ja dann anschließend mit dem Geld zumindest sich eine Überbrückung schaffen. Ich halte es schon für sinnvoll. Also, ich habe das Geld, glaube ich, sinnvoll eingesetzt." (I.03.W.nk)

Eine Person der Gruppe „Hausfrauen“ legte das Übergangsgeld für die Zukunft an:

„Ich habe das [Übergangsgeld] zunächst mal genutzt, um diese Auszeit zu nehmen. Ich habe es auch angelegt, was man so macht, also um sich ein Stück weit abzusichern. In der Politik ist man ja tatsächlich auf Gedeih und Verderb der Wahl unterworfen. Man sitzt auf dem heißen Stuhl, immer irgendwie. .. Ich habe es damals für eine Wohnung angelegt, weil ich gedacht habe, das könnte mal was sein für die Zukunft." (I.07.O.nk)

Eine andere Person dieser Gruppe verwendete es für den Ausbau ihres Hauses:

„Ich habe mein Arbeitszimmer auf dem Boden ausgebaut. ... Für mich war es unbedingt sinnvoll. Ich brauchte nach der Zeit einfach einen eigenen Raum, als gute Hausfrau und Mutter hatte ich natürlich keinen Raum. Das war mir einfach wichtig, ein eigenes Arbeitszimmer zu haben, diese Selbständigkeit zu wahren. Es ist ja eine ganz schöne Umstellung.“ (I.11.W.nk)

14 Dies würde theoretisch auch für Einkommen aus der Privatwirtschaft gelten, wenn sie ab Antritt der Arbeitsstelle der Bundestagsverwaltung gemeldet würden. 
Obwohl die Mehrzahl der Befragten das Übergangsgeld für den eigentlichen Zweck nicht gebraucht hat, waren sich alle in der Ansicht einig, dass es für ausgeschiedene Bundestagsabgeordnete eine Versorgungsregelung geben müsse, insbesondere für diejenigen, die keine Rückkehrmöglichkeit hätten und sich beruflich neu etablieren müssten. So meinte eine Person, die unmittelbar nach dem Mandatsverlust wieder eine Anstellung gefunden hatte:

„[F]ür andere, die nicht diesen schnellen Übergang ins Berufsleben hatten - es gibt welche, die sind arbeitslos gewesen lange Zeit - da ist das schon eine wirtschaftliche Absicherung, dass man nicht ganz mittellos dasteht. Denn von den Diäten, die man als Bundestagsabgeordneter bekommt, da bleibt nichts übrig, was man da sparen könnte. Da hängen so viele Blutsauger dran, ein Teil davon ist ja ohnehin nur eine Aufwandsentschädigung für das Büro, das man im Wahlkreis unterhalten muss, die ganzen Büroausgaben, die da anfallen. Ich habe sehr sparsam gelebt, habe auch keine hohe Miete bezahlt, es hat gerade so gereicht. Von dem, was man eigentlich als Gehalt bekommt, da darf man so viele Spenden machen und man muss ja auch den nächsten Wahlkampf daraus finanzieren. Sicherlich kann man auch Spenden dafür einwerben, das habe ich auch gemacht, aber da ist alles draufgegangen. Ich glaube der Wahlkampf '94, der hat mich damals 35.000 DM gekostet und hat zu nichts geführt für mich persönlich. Ich habe die Abrechnung, die hebe ich mir fein säuberlich auf, die habe ich noch da. Was dann noch übrig geblieben ist, habe ich an die Kreisverbände überwiesen als Spende." (I.15.O.k)

Ein anderer ehemaliger Abgeordneter betonte, dass das Übergangsgeld insbesondere für Parlamentarier nötig sei, die nicht wieder gewählt wurden:

„Ja, die Übergangsgelder, die braucht man. Ich meine, schön, ich hatte mich natürlich vorher gekümmert, was ich hinterher mache, wenn man es weiß, dass man ausscheidet. Aber da sind sicherlich eine Reihe von Kollegen, die es nicht wissen, wenn sie ausscheiden, sondern wo einfach der Wähler sagt: ,Ja, ich will Dich nicht mehr'. Und man steht dann auf der Straße und muss zum Arbeitsamt gehen, das ist sicherlich nicht toll." (I.25.O.nk)

Manche Befragte vertraten die Ansicht, dass die öffentlich Bediensteten von der Versorgungsregelung des Übergangsgeldes ausgenommen werden sollten. Die Untersuchungsergebnisse zeigen allerdings, dass auch die Beamten und Angestellten des öffentlichen Dienstes das Übergangsgeld nutzten, um sich beruflich neu zu etablieren, weil sie nicht wieder zu ihrer vorherigen Dienststelle zurückkehren wollten.

Hans Herbert von Arnim ist der Ansicht, dass Berufspolitiker nach ihrem Ausscheiden aus der Politik überversorgt seien und behauptet: „Da viele Berufspolitiker schon nach wenigen Jahren eine hohe Versorgung erwerben, die meist lange vor der üblichen Altersgrenze fällig wird, ist es ihnen ein leichtes, im vorzeitigen Ruhestand noch einen Zweit- oder Drittberuf zu ergreifen und daraus weitere Einkommen zu erzielen. " ${ }^{15}$ An späterer Stelle heißt es: „Die Aussage, daß Politiker überversorgt sind, gilt auch und erst recht für Übergangsgelder." ${ }^{16}$ Diese verallgemeinernde Aussage lässt sich anhand der quantitativen Erhebungen in der Studie widerlegen: Von den insgesamt 368 ausgeschiedenen Abgeordneten

15 Hans Herbert von Arnim, Diener vieler Herren. Die Doppel- und Dreifachversorgung von Politikern, München 1998, S. 127.

16 Ebenda, S. 132. An anderer Stelle formuliert von Arnim: „Eindeutig ist auch, daß Minister, Parlamentarische Staatssekretäre und Abgeordnete durch aberwitzige Übergangsgelder und Altersversorgungen weit überversorgt sind. " Hans Herbert von Arnim, Fetter Bauch regiert nicht gern. Die politische Klasse - selbstbezogen und abgehoben, München 1997, S. 92. 
der 12. und 13. Wahlperiode haben 270 Personen einen Versorgungsanspruch erworben und 98 Personen nicht, weil sie weniger als acht Jahre im Bundestag tätig waren. Nach acht Jahren Parlamentstätigkeit ${ }^{17}$ liegt das Pensionsalter bei 65 Jahren und sinkt mit jedem weiteren Parlamentsjahr um ein Jahr bis zu einem Mindestalter von 55 Jahren (nach 18 Parlamentsjahren). ${ }^{18}$

Überprüft man die Lage der ausgeschiedenen Bundestagsabgeordneten der 12. und 13. Wahlperiode, dann stellt man fest, dass von den 368 ausgeschiedenen Abgeordneten 156 im Pensionsalter und 212 nicht im Pensionsalter ausgeschieden sind. Also rund 58 Prozent der ausgeschiedenen Abgeordneten dieser beiden Wahlperioden mussten beziehungsweise müssen noch die Zeit bis zum Pensions- oder Rentenalter durch andere Einkommensquellen überbrücken. Für die ausgeschiedenen Abgeordneten der 9. und 10. Wahlperiode stellte Heino Kaack fest: „Nach dem gegenwärtigen Stand der Altersversorgung muß also konstatiert werden, daß nur eine Minderheit der Abgeordneten über eine sofort wirksame finanzielle Absicherung verfügt. [...] Eine nicht unerhebliche Anzahl von Parlamentariern hat nach wie vor Absicherungsprobleme. " ${ }^{19}$ Und etwa jeder dritte Ausgeschiedene hatte nach seinen Daten keinerlei Pensionsansprüche erworben. ${ }^{20}$

In einigen Fällen kann durch „Streckung“ des Übergangsgeldes die Lücke zwischen Mandatsende und Altersversorgungsbeginn überbrückt werden:

„Ich habe mir das [Übergangsgeld] monatlich auszahlen lassen, um diese Zeit zu überbrücken. Denn wenn ich gewusst hätte, was das für ein Theater wird mit der Frühpensionierung - da stand ich ja zunächst ohne Einkommen da ab '98. Da war mir das Übergangsgeld ganz recht. Das hat mich also abgefedert." (I.23.W.nk)

Ebenso eine Person der Gruppe „Hausfrauen“, deren Ehepartner verstorben war:

„Ich hatte zum einen meine Witwenrente ... und hab’ dann dies Übergangsgeld, das ich bekommen habe, bis ich dann selber überhaupt meine Altersruhe bekomme, habe ich das Übergangsgeld gesplittet." (I.28.W.nk)

Die verallgemeinernde Behauptung, dass Berufspolitiker durch Übergangsgelder überversorgt seien, ist nach den dargelegten Befunden - zumindest für Abgeordnete, welche die Masse der Berufspolitiker stellen - sicher nicht haltbar.

\subsection{Die Rolle der Parteien beim beruflichen Wiedereinstieg}

Die Rolle der Parteien beim beruflichen Wiedereinstieg wurde von nahezu allen Befragten negativ beurteilt. Insbesondere jenen, die ihr Mandat durch eine Wahlniederlage verloren und ein Stellenangebot ihrer Partei begrüßt hätten, begegneten die Parteien mit unverbindlichen Ankündigungen und Gleichgültigkeit. Beispielsweise haben Parteikollegen der folgenden befragten Person nach deren Mandatsverlust bekundet, auf jeden Fall helfen zu wollen, was aber letztlich ohne praktische Folgen blieb:

17 Aufgrund von Rundungsvorschriften reicht eine tatsächliche Mitgliedschaft von sieben Jahren und 183 Tagen aus. Vgl. Deutscher Bundestag - Verwaltung (Hrsg.), a.a.O., S. 12.

18 Vgl. ebenda, S. 13.

19 Heino Kaack, Zur Abgeordnetensoziologie des Deutschen Bundestages: Zugehörigkeitsdauer und Altersschichtung, in: ZParl, 19. Jg. (1988), H. 2, S. 169 - 187, S. 183.

20 Vgl. ebenda, S. 181. 
„Erst mal aus dem Gefühl, aus der Stimmung heraus [sagen die Parteikollegen]: ,Wir sind für Dich da' und so was, aber dann passiert nicht viel. Da waren nicht irgendwo Angebote oder so, in Aufsichtsrat reinkommen oder so was, das war nicht. Und hier hat auch keiner gewartet bei der Kommunalpolitik nach dem Motto: ,Toll, wir haben wieder einen guten Mann hier bei uns'. Und im Kreistag: ,Was können wir für Dich tun? Welchen Posten können wir Dir jetzt hier geben, Du hast ja Zeit jetzt' oder so, da war nichts. Da muss man wieder richtig von vorne [anfangen]. Man hat zwar Freunde oder so, aber die geben keinen eigenen Ausschusssitz deshalb her, das steht ja nicht zur Debatte.“ (I.14.W.k)

Andere fassten die Rolle ihrer Partei nach dem Mandatsverlust wie folgt zusammen:

„Die eigene Partei hat sich einen feuchten Kehricht um mich gekümmert.“ (I.31.O.k)

„Nee, ich habe das [mit dem beruflichen Wiedereinstieg] selber gemacht alles. Wissen Sie, das ist immer so, wenn Du dabei bist, wenn Du zu den Mächtigen mitzählst, dann bist Du einer, der nach der Devise geht: ,Ohne mich geht es nicht'. Aber wenn Du mal nicht mehr zum Kreis der Mächtigen dazu gehörst, dann sind Sie plötzlich aus den Augen aus dem Sinn bei vielen. Das ist leider so." (I.34.W.k)

Hingegen wurden Befragte, die freiwillig auf ihr Mandat verzichtet hatten und sich weitgehend aus der aktiven Politik zurückziehen wollten, von ihrer Partei ausdrücklich gedrängt, für andere politische Ämter zu kandidieren. Dies hinderte die Betroffenen zum Teil sogar daran, außerhalb der Politik eine Erwerbsarbeit anzunehmen. So konnten die folgenden Befragten dieser Bedrängnis nicht standhalten, obwohl sie beteuerten, wirklich keine Lust mehr auf ein anderes politisches Amt gehabt zu haben:

„[Nach dem Bundestagsmandat] wurde ich dann Staatssekretär ... . Es kam ein bisschen überraschend, eigentlich wollte ich gar nicht mehr, ich bin ja freiwillig ausgeschieden. Die wollten mich damals alle halten." (I.08.W.nk)

„Dann wird man von Mal zu Mal [für andere politische Ämter] gefragt: ,Ach, willst Du es nicht noch einmal machen?' Dann fängt man an weich zu werden und fühlt sich auch gebauchpinselt, ist eitel genug, um das anzunehmen. Ob das immer ehrlich gemeint war, weiß ich nicht. [...] Die einen sagen: ,Der ist so bekannt, vielleicht wählen sie den auch wirklich im Gegensatz zu jedem anderen, der nicht so bekannt ist'. Da hat es sicherlich unterschiedliche Gründe dafür gegeben, dass die Partei mich gebeten hat zu kandidieren. ... Von mir aus, ich habe mich nicht gemeldet, sondern die kamen auf mich zu ... . Ob mich nun alle geliebt haben, das weiß ich nicht, aber zumindest sahen sie eine Chance, auf diese Art und Weise eine Mehrheit zu bekommen, das ist auch gelungen." (I.18.W.nk)

Damit decken die Untersuchungsergebnisse eine auf den ersten Blick absurde Situation auf, nämlich dass diejenigen, die ihr berufspolitisches Engagement fortsetzen wollten, von den Parteien ignoriert wurden, wohingegen diejenigen, die ihre berufspolitische Tätigkeit aufgegeben hatten, von den Parteien nahezu massiv gedrängt wurden, ihr politisches Engagement wenigstens auf einer anderen Ebene fortzusetzen. Dies lässt sich mit der Art des Ausscheidens der entsprechenden Befragten erklären: Die Mandatsverlierer (die vorwiegend von ihrer Partei ignoriert wurden) hatten offenbar weder bei der Parteispitze noch bei der Parteibasis noch bei den Wählern entsprechend Rückhalt gefunden, um ihr Mandat halten zu können. Hingegen waren die von der Partei Umworbenen alle aus einer stabilen politischen Position freiwillig aus persönlichen Gründen ausgeschieden und besaßen deshalb nach wie vor das Potential, auch für andere Ämter gewählt zu werden. Als „Wählerstimmenmagnete“ konnten sie der Partei noch zu politischem Einfluss verhelfen. Die Mandatsverlierer waren allerdings nicht durchweg uninteressant für ihre Partei. Wenn es um die 
Besetzung politischer Stellen in öffentlichen Institutionen ging, hatten auch sie eine Chance, angefragt zu werden, sofern sie während ihrer Mandatszeit durch besondere Fachkompetenzen und herausragende Leistungen auf dem entsprechenden Gebiet aufgefallen und bei den entsprechenden Stellen bekannt waren.

Hans Herbert von Arnim geht davon aus, dass die „politische Klasse“ besonders bemüht sei, die ausgeschiedenen Kolleginnen und Kollegen irgendwo unterzubringen. ${ }^{21}$ Die interne Postenvergabe in der Politik stellt für ihn ein „Zweites System“ dar, das hinter dem offiziellen errichtet wurde. ${ }^{22}$ Doch die folgende Erfahrung einer befragten Person mit der Presse stellt die Vertrauenswürdigkeit solcher Meinungen in Frage:

„Nichtsdestotrotz fand sich ein Artikel in fast allen bundesdeutschen Blättern, .. in dem es hieß: , .. weicher Fall nach Brüssel: hoch dotierter Spitzenposten auf europäischer Ebene.' Das war das allerletzte. Und wenn so etwas erst mal in der Zeitung steht, dann bist Du fertig. Dann braucht man sich um nichts anderes mehr zu bewerben, weil jeder glaubt, die ist ja untergekommen. Ich habe dann diesen Journalisten zur Rede gestellt und ihn um ein Gespräch gebeten und gesagt: ,Sie haben mir was angetan. Wenn das so läuft, wenn Journalismus so abgeht, dass überhaupt nicht mit der Betroffenen gesprochen wird, gar nicht recherchiert wird, der allergrößte Unsinn geschrieben wird. Ich habe 4.000 Mark Außenstände, das sind meine eigenen Reisekosten der letzten vier Wochen, und Sie behaupten hier, ich hätte einen hoch dotierten Spitzenposten. Das ist ein Ehrenamt'." (I.29.W.nk)

Die vorliegenden Untersuchungsergebnisse legen nicht im Geringsten nahe, dass die Parteien über ein verlässliches Netz von Versorgungsposten für ausgeschiedene Berufspolitiker verfügen, geschweige denn, dass sie im Begriff wären, ein solches auf- oder auszubauen. Im Gegenteil vermitteln die Ergebnisse eher den Eindruck, dass sich die Parteien für den Großteil ihrer ausgeschiedenen Abgeordneten nicht mehr interessieren.

\subsection{Wirtschaftliche Notlagen nach dem Mandatsverlust}

Manche Befragte gerieten nach dem Mandatsende in eine wirtschaftliche Notlage. Alle diese „Problemfälle“ waren unbeabsichtigt ausgeschieden, hatten ihr Mandat durch eine Wahlniederlage verloren. Keiner von ihnen hatte eine Rückkehrmöglichkeit zur vorherigen Stelle, und keiner hatte bereits das Pensions- beziehungsweise Rentenalter erreicht, so dass sich die Betroffenen um eine Erwerbsmöglichkeit bemühen mussten.

„Mir war klar, als ich aus dem Bundestag raus bin, dass ich sofort wieder eine Erwerbsarbeit aufnehmen musste, um überhaupt wieder in diese sozialen Sicherungssysteme rein zu kommen." (I.30.W.k)

Diese befragte Person hatte zunächst durch eine Initiativbewerbung eine befristete Arbeitsstelle bekommen. Allerdings wurde ihr Vertrag nicht verlängert:

„Nach dem befristeten Vertrag bin ich regulär erwerbsarbeitslos geworden mit den ganzen materiellen Katastrophen, die da folgen. Dazu ist im Übrigen auch noch zu sagen, ein Umstand, der den meisten Menschen nicht bewusst ist, weil man als Abgeordneter auch ständig unter so einem Reichtumsverdacht steht oder unter so einem Überversorgungsvorbehalt ... .

21 Hans Herbert von Arnim, Fetter Bauch, a.a.O., S. 99, vgl. auch S. 73.

22 Vgl. ders., Das System. Die Machenschaften der Macht, München 2001, S. 48. 
Wenn man einmal nur drin war, dann hat man sicherlich eine gewisse Zeit lang dieses Übergangsgeld, aber dafür hat man diese unglaubliche Steuerlast zu schleifen, als Abgeordnete [einer kleinen Partei] müssen Sie ungeheuer viel Geld an die Partei abführen. Es ist ein materielles Desaster. ... Aber wenn Sie kein Geld mehr kriegen, dann fallen Sie der Sozialhilfe anheim, weil Sie keinen Arbeitslosenbeitrag im Parlament bezahlen. Das reflektiert die Öffentlichkeit aber nicht. Das versteht auch keiner. Sie können mit kaum einem anderen Menschen überhaupt über ihre Verhältnisse reden. Sie müssen immer erst erklären, wie das ist und das fruchtet irgendwie nicht, weil, wenn sie die Realität dann hören, diese so unglaublich ist, so kontrastiert mit dem, was sie in der Bildzeitung lesen, dass sie es nicht glauben wollen. Also, man ist da sehr einsam damit. Sie müssen das alles alleine durchziehen." (I.30.W.k)

Die Gründe für die Schwierigkeiten, auf dem Arbeitsmarkt eine Anstellung zu bekommen, lagen unter anderem an ihrer Überqualifikation für Positionen auf mittlerer Ebene.

„Ich habe ja nicht das Problem, dass ich nichts kann, sondern ich bin überqualifiziert. Ich kann ja praktisch von jedem den Job machen. [...] In einem Bewerbungsgespräch habe ich einen ziemlich großen Auftritt hingelegt, aber immer so, dass sie [die Vorstandsvorsitzende] entscheiden muss, also schon ihr den Raum gelassen, dass sie ja nicht meint, ich wollte ihren Job machen. Also, ich hatte alles sehr gut geplant und spielerisch vom Auftritt her habe ich das gut hingekriegt. Und dann kriegte ich einen Telefonanruf: ,Sie waren überragend, Sie waren mit Abstand die Beste überhaupt, wir nehmen Sie deshalb nicht. Wir stellen jemand ein, der in drei Jahren oder nie so weit sein wird wie Sie, weil wir diese Person stärker an unsere Institution binden möchten'“" (I.30.W.k)

Oder eine andere Person der Gruppe „Problemfälle“ erklärte:

„Nun ist das Schlimme, man kommt ja dann auch finanziell an Grenzen, ... wenn man arbeitslos geworden ist. Da nimmt man ja eigentlich innerlich jeden Job an. Aber ich kann eben aufgrund meiner Qualifikation und meines politischen Werdegangs mich nicht als Kraftfahrer bewerben. Das ist ein Fluch auf der anderen Seite, ich kann mich nicht für jeden Job bewerben. Das geht ab Abteilungsleiter oder Geschäftsführer, auf der Ebene eines kleineren Bereiches erst los, sonst sagt jeder: ,Der muss doch irgendwas verbrochen haben, wenn er sich mit der Qualifikation um diese Stelle bewirbt'. Ich habe also nur eine ganz schmale Bandbreite an Stellen, die überhaupt in der Öffentlichkeit angeboten werden, die überhaupt für mich in Frage kämen, weil ich für alles andere überqualifiziert bin. Obwohl ich mich um alles Mögliche beworben habe." (I.31.O.k)

Auch die Parteizugehörigkeit wirkte sich bei den Betroffenen negativ auf eine Anstellung aus. Insbesondere bei Stellen im politischen Bereich spielte diese eine Rolle. In der Privatwirtschaft scheuten sich Arbeitgeber zum Teil, Befragte bestimmter Parteien (Bündnis 90/ Die Grünen oder PDS) einzustellen. Die zeitweilige Arbeitslosigkeit hatte für die Betroffenen nicht nur materiell negative Konsequenzen, sondern auch in psychischer Hinsicht. Sie mussten den Absturz vom privilegierten hofierten Bundestagsabgeordneten zum sozial ausgegrenzten Arbeitslosen verkraften. Die zwei obigen befragten Personen schilderten ihre Lage folgendermaßen:

„Also, Urlaub ist seit fünf Jahren sowieso gestrichen, ich verzichte auf alles, was irgendwie Geburtstagsfeiern, das fällt alles flach, bloß um das Haus noch einigermaßen finanzieren zu können. Und da darf irgendwie nichts dazwischen kommen. So eine Beule am Auto, die muss bleiben. [...] Sie merken auch ein Stück Verbitterung, die sich dann einstellt, wenn man fünf Jahre lang wirklich kaum noch ruhig schlafen kann und jeden Abend im Bett Kassensturz macht und zu keinem anderen Ergebnis kommt. Das belastet einen schon und hat auch Einfluss aufs Wesen, es verändert einen, man verliert also auch ein Stück weit Persönlichkeit, Frohnatur, die man mal hatte, das ist alles weg." (I.31.O.k) 
„Diese ganzen sozialen Abstiegskränkungen, die man durchläuft, sind zwar aus der Sicht anderer wattiert, aber relativ zu dem, was ich mal hatte, doch schwierig zu verdauen. [...] Also, da gibt es ungeheure Krisen, und Sie brauchen ein ungeheures Maß an Durchhaltevermögen, vor allen Dingen an psychologischen Bewältigungsmechanismen. [...] Und das menschlich Schlimmste ist, dass man dann so eine Angstgestalt wird und anderen aus der Fraktion einen Spiegel vorhält von der sozialen Fragilität ihres Postens, der mit dem, was sie sich zusammengebaut haben an Selbstwert und Wichtigkeit so furchtbar kontrastiert." (I.30.W.k)

\section{Politischer Verbleib der Befragten}

Die Untersuchungsergebnisse zum politischen Verbleib der Befragten zeigen, dass die große Mehrheit der Befragten (26 von 38) ihre politische Aktivität nach dem Ausscheiden aus dem Bundestag fortgesetzt hat. Dabei ist es unerheblich, ob sie freiwillig aus dem Bundestag ausgeschieden sind oder abgewählt wurden. Der Hauptgrund für die Fortsetzung der politischen Aktivität war bei den meisten, dass sie parallel zum Bundestagsmandat noch weitere politische Ämter innehatten, deren Amtszeiten noch nicht zu Ende waren. Zu solchen Ämtern zählen der Parteivorsitz, die Mitgliedschaft in Parteivorständen auf Kreis-, Bezirks-, Landes- oder Bundesebene sowie Kreistagsmandate, Gemeinderatsmitgliedschaften, das Amt des Landrates oder des ehrenamtlichen Bürgermeisters. Oft hatten einzelne Personen mehrere Ämter inne, insbesondere einen Parteivorsitz in Kombination mit einem Mandat. Insgesamt bekleideten von den 18 Personen, deren politisches Engagement sich nahtlos fortsetzte, elf neben dem Bundestagsmandat noch ein zusätzliches Mandat auf Kommunal- oder Kreisebene. Bei Neuwahlen für diese Positionen kandidierten die meisten erneut. Manche bewarben sich sogar um weitere Mandate auf unterer Ebene, was durchweg erfolgreich war. Dagegen waren die Versuche, für den Bundestag oder das Europäische Parlament zu kandidieren, allesamt erfolglos. Die entsprechenden Befragten hatten dafür von ihrer Partei keine Unterstützung erhalten, scheiterten bereits an der Nominierung für aussichtsreiche Listenplätze. Den Grund für die mangelnde Unterstützung sahen die Befragten darin, sich nach dem Verlust des Bundestagsmandates zu wenig für die Parteipolitik engagiert zu haben:

„Erst nach vier Jahren ging ich wieder zu den politischen Versammlungen und habe dann auch für Europa kandidiert, allerdings war es zu kurzfristig. ... Ich wurde auf einem aussichtslosen Platz nominiert.“ (I.37.W.k)

Eine andere Person resümierte ihren gescheiterten Versuch zur Europakandidatur:

„Ja, woran ist das gescheitert? Ich habe mich in den vier Jahren wirklich nur um meinen Betrieb gekümmert und nicht mehr ... um Parteibelange. Man hätte vielleicht aktiver in dieser Zeit ... sein müssen. Ich hätte da sicher mehr auftreten müssen, vielleicht auch ab und zu mal was für irgendwelche Zeitungen, Zeitschriften schreiben müssen, damit man im Gespräch bleibt. Und ja, parteiinterne Intrigen, die da auch eine Rolle spielen bei solchen Sachen, bei solchen Aufstellungen. [...] Da gab's natürlich genug Leute, die gesagt haben: ,Der kann vielleicht kandidieren, aber irgendwo ganz hinten.' Und dafür war nun wiederum ich mir zu schade, dass ich gesagt habe: ,Wenn ich schon kandidiere, dann kandidiere ich auf einem Listenplatz, der erfolgreich ist. Also, ich mach' mich nicht irgendwo zum Popanz dafür, dass ich schon mal [im Bundestag] drinne war." (I.04.O.nk) 
Eine weitere Person berichtete:

„Ich habe auf der Landesversammlung keine Mehrheit hinter mich gebracht, sondern bin einem .. 24-jährigen Studenten unterlegen, weil es auch damals hieß, es sollen jetzt auch mal die Jungen vor, so dass ich das auch nicht weiter betrieben habe, nicht weiter versucht habe. Wenn das Votum der Landesversammlung so war, habe ich das akzeptiert." (I.16.W.k)

Zwölf der 38 Befragten beendeten ihr politisches Engagement nach dem Ausscheiden aus dem Bundestag. Ein Grund dafür waren Differenzen in der Partei, die sich während der Mandatszeit entwickelt hatten und eine damit einhergehende Entfremdung:

„Nee, Politik an und für sich macht mir Spaß, ... aber Politik in dieser Partei hat mir keinen Spaß mehr gemacht oder passte nicht mehr mit dem überein, was sich so entwickelt hat auf beiden Seiten: In mir hat sich erst mal etwas entwickelt durch die Erkenntnisse und den neuen Umgang, den man hatte und die Partei hat sich auch entwickelt und beides hat sich aber nicht in die gleiche Richtung entwickelt. [...] Ich bin dann nach zwei Jahren ganz raus aus der Partei." (I.04.O.nk)

Weitere Gründe liegen im beruflichen Engagement nach dem Bundestagsmandat, welches für aktive Politik wenig Zeit ließ. So wollte sich ein Befragter entweder richtig in die Politik einbringen oder gar nicht:

„.. ich konzentriere mich jetzt auf das Leben nach der Politik und außerhalb der Politik und - ein bisschen schwanger kannst du nie sein: entweder du bist dabei oder du bist nicht dabei." (I.34.W.k)

Eine Person hatte den Eindruck, dass die Partei sich nicht mehr für sie und ihre erworbenen Erfahrungen interessierte und dass dies ein generelles Problem ausgeschiedener Abgeordneter sei:

„Ich habe mich aus der gesamten parteipolitischen Arbeit ausgeklinkt. Das ist auch nicht gefragt. Was mich eigentlich immer wieder erstaunt, auch im kommunalpolitischen und landespolitischen Bereich, dass die Erfahrung von altgedienten Politikern, dass das nicht aufgenommen wird, nicht umgesetzt wird. Nicht dass Sie das Gefühl haben, ich habe mich beleidigt zurückgezogen, weil mich keiner gefragt hat, das meine ich nicht, sondern dass man nicht ganz bewusst die Erfahrung der Politiker ausnutzt, die engagiert irgendwo drin waren. Die Einzigen, die das genutzt haben, das waren [Unternehmen], die das insofern ausnutzen, bis heute hin, indem sie sagen: ,Du hast den Bundestagsausweis noch, Du hast hier Kontakte, da Kontakte', das nutzen die dann aus, wenn man von ,Ausnutzen' sprechen kann. Sie haben oftmals das Problem, an einen Bürgermeister heranzukommen, Landrat oder in einem Ministerium." (I.20.W.k)

Die große Mehrheit der Befragten wurde nach dem Mandatsende nicht mehr von Interessengruppen in Anspruch genommen. Nur noch wenige der Befragten, die weiterhin ein politisches Amt auf unterer Ebene wahrnahmen, wurden noch von örtlichen Interessengruppen konsultiert. In ganz wenigen Fällen fand eine Beeinflussung auf politische Entscheidungen auf Bundesebene statt. Dabei zeigten sich zwei Formen von Lobbyismus: zum einen auf der Basis eines Angestelltenverhältnisses bei einem bestimmten Interessenverband und zum anderen auf der Basis als selbständiger Berater. Eine befragte Person, die nach ihrem Bundestagsmandat auf Angestelltenbasis die Geschäftsführung eines Bundesverbandes einer Branche übernahm, hatte vorwiegend die Aufgabe, für den Verband als Lobbyist zu arbeiten:

„Dann bekam ich am nächsten Morgen einen Anruf vom Bundesverband .. : , Unser Leiter der Geschäftsstelle .. wird uns verlassen. Hätten Sie nicht Interesse an dieser Position? Wir haben gestern erfahren, dass Sie noch keine weitere Position eingenommen haben, wäre das 
etwas für Sie?' Und ich: ,Ja, ich glaube schon, dass ich das kann.' Und so wechselte ich auf die andere Seite. ... Es war für mich sehr anstrengend, hatte sehr viel Neuland zu betreten, hat allerdings auch sehr viel Spaß gemacht, mal wieder etwas Neues hinzuzulernen. ... Es fiel mir nicht schwer, mit den Verantwortlichen der [Fachpolitik] weiterhin vertrauensvoll zusammen zu arbeiten, auch von der anderen Seite aus, weil sie mich ja kennen gelernt hatten als jemand, auf dessen Wort man etwas geben kann. Und wenn ich also nun nachfragte: ,Wie sieht die Gesetzgebung aus, was habt Ihr vor? Da haben wir aber erhebliche Einwände von Seiten der .. Industrie vor allen Dingen der kleinen Hersteller .., die herunterzufallen drohen. Wenn Ihr die jetzt vom Markt werft, dann steht der Bevölkerung das und das und das Produkt nicht mehr zur Verfügung'. Also, es hat mich weder geniert, noch war's mir peinlich, noch habe ich Skrupel gehabt, diese Aufgabe zu übernehmen, weil ich wusste, wie ich sie übernehmen würde und wie ich mit meinen ehemaligen Kolleginnen und Kollegen umgehen würde und dass sie sich auf mein Wort genauso verlassen können, wie damals auch. Insofern fiel es mir sehr, sehr leicht, diese Aufgabe zu übernehmen." (I.29.W.nk)

Diejenigen, die sich als Unternehmensberater selbständig machten, wurden weiterhin von Vertretern der Wirtschaft konsultiert. Ob es sich um dieselben wie zur Bundestagszeit handelte, hing davon ab, auf welcher Ebene die Unternehmensberatung stattfand. Eine befragte Person gab an, dass der Lobbyismus in Form selbständiger Berater zunehmend systematisch organisiert würde. Dieser Aspekt konnte im Rahmen dieser Arbeit nicht weiter verfolgt werden, verdient aber politikwissenschaftliche Aufmerksamkeit.

\section{Forschungsausblick}

Die hier dokumentierten Daten, Fakten und Einschätzungen zum beruflichen und politischen Verbleib ehemaliger Bundestagsabgeordneter sind nicht repräsentativ. Sie vermitteln aber ein realistisches Bild der beruflichen und politischen Situation ausgeschiedener Berufspolitiker. Diese zeigen, dass mit dem Amt auf Zeit Risiken für den nachpolitischen Lebensweg verbunden sind.

(1) Eine politikwissenschaftlich bedeutende Frage ist, an welcher Stelle des politischen Systems diese Risiken Auswirkungen haben. Die hier vorgelegten Ergebnisse führen zu der Vermutung, dass der Einfluss weniger im Parlament als vielmehr im vorparlamentarischen Rekrutierungsbereich zu suchen ist. Eine neu zu untersuchende These lautet, dass die Abgangsbedingungen den Zugang zum politischen Amt bestimmen und damit das von Mandatsaspiranten vorhergesehene und als unakzeptabel beurteilte Risiko des existentiellen Scheiterns wesentlich für die berufssoziologische Zusammensetzung des Bundestages mitverantwortlich ist. Dies ergibt eine neue Erklärungsperspektive innerhalb der Diskussion über die zunehmende Verbeamtung der Parlamente.

(2) Aus dem Ergebnis, dass die wenigsten der Befragten nach dem Mandatsende in ihren vorherigen Beruf zurückgekehrt sind, sondern die meisten ihren Beruf gewechselt haben, ergibt sich die Frage, welches die typischen Berufsfelder sind, in die die ehemaligen Bundestagsabgeordneten wechseln. Die Beraterberufe scheinen eines zu sein. Hier wäre zu erforschen, ob ehemalige Berufspolitiker in diesen typischen Berufsfeldern organisierte Netzwerke bilden, die Einfluss auf Entscheidungsprozesse innerhalb des politischen Systems nehmen.

(3) Die Tatsachen, dass es ehemalige Bundestagsabgeordnete gibt, die nach dem Mandatsverlust zu Sozialhilfeempfängern werden, dass die Parteien offenbar keine verlässlichen 
Auffangnetze für ausgeschiedene Mandatsträger haben und dass das Übergangsgeld kaum seinem eigentlichen Zweck dient, könnten einen Impuls für die Diätendebatte geben, die unter dem Gesichtspunkt dieser Bedingungen der parlamentarischen Nachversorgung ganz anders geführt werden müsste. Zum Beispiel könnte darüber nachgedacht werden, ob Bundestagsabgeordnete in die sozialen Sicherungssysteme einzahlen, um nach dem Mandatsverlust einen Versorgungsschutz zu haben. Oder die Diäten könnten erhöht werden, um Mitgliedern des Bundestags den finanziellen Spielraum zu ermöglichen, sich für die nachparlamentarische Zeit privat zu versichern. Eine veränderte Versorgungsregelung könnte wiederum Auswirkungen auf die Rekrutierung des parlamentarischen Personals haben.

(4) Von enormer Bedeutung wäre zudem, den Verbleib ehemaliger Abgeordneter international vergleichend zu erforschen. Wie sind die rechtlichen Regelungen der Nachversorgung in den einzelnen Ländern gestaltet? Im Schweizer Milizsystem zum Beispiel ist ein reines Berufspolitikerdasein nicht vorgesehen. Wie vereinbaren Bundespolitiker in der Schweiz Beruf und Mandat? Wie schlägt sich das Milizprinzip in der rechtlichen Ausgestaltung politischer Ämter bis hin zur Nachversorgung nieder? In Großbritannien dürfen Angehörige des öffentlichen Dienstes nicht ins Parlament gewählt werden. Im Bundestag hingegen liegt der Anteil der Beamten und Angestellten des öffentlichen Dienstes bei knapp 45 Prozent der Abgeordneten. Diese Gruppe ist hinsichtlich der parlamentarischen Nachversorgung keinerlei Risiko ausgesetzt. Wie sieht die berufssoziologische Zusammensetzung des britischen Parlaments ohne Angehörige des öffentlichen Dienstes aus? Wie ist die Nachversorgung dort geregelt, und gibt es „Reaktionen“ der Abgeordneten oder der Parteien auf das Risiko einer gescheiterten Wiederwahl?

\section{Der „Hammelsprung“ im Deutschen Bundestag}

Kai Zähle

Kaum eine parlamentarische Übung erfreut sich so großer allgemeiner öffentlicher Aufmerksamkeit wie der so genannte Hammelsprung. Dies liegt nicht zuletzt an der außergewöhnlichen Bezeichnung dieses Abstimmungsverfahrens, welches in $₫ 51$ Abs. 2 der Geschäftsordnung des Bundestages (GO BT) geregelt ist: „Nachdem die Mitglieder des Bundestages auf Aufforderung des Präsidenten den Sitzungssaal verlassen haben, werden die Türen bis auf drei Abstimmungstüren geschlossen. An jeder dieser Türen stellen sich zwei Schriftführer auf. Auf ein Zeichen des Präsidenten betreten die Mitglieder des Bundestages durch die mit ,Ja', ,Nein’ oder ,Enthaltung' bezeichnete Tür wieder den Sitzungssaal und werden von den Schriftführern laut gezählt.“ Der Begriff „Hammelsprung“ bedeutet in der Parlamentspraxis der Bundesrepublik also die Auszählung der Stimmen

1 Peter Schindler, Datenhandbuch zur Geschichte des Deutschen Bundestages 1983-1991, BadenBaden 1994, S. 576, S. 581. 